\title{
THE RELATIONSHIP BETWEEN CHRONIC MUSCULOSKELETAL PAIN, QUALITY OF LIFE AND SARCOPENIA
}

Turkish Journal of Geriatrics

DOI: 10.31086/tigeri.2021.200

2021; $24(1): 60-70$

\section{- Fulya BAKILAN ${ }^{1}$ \\ - Merih ÖZGEN ${ }^{2}$ \\ - Burcu ORTANCA ${ }^{1}$ \\ - Ayse EKIM ${ }^{1}$ \\ - Onur ARMAĞAN² \\ - Funda TAŞÇIOĞLU BERKAN² \\ - Fezan ŞAHIN MUTLU ${ }^{3}$}

CORRESPONDANCE

\section{${ }^{1}$ Fulya BAKILAN}

Eskisehir City Hospital, Physical Medicine and

Rehabilitation, Eskişehir, Turkey

\section{Phone: +905057737335 \\ e-mail: fulyabakilan@gmail.com}

Received: Oct 26, 2020

Accepted: Feb 22, 2021

Eskisehir City Hospital, Physical Medicine and Rehabilitation, Eskişehir, Turkey

2 Eskisehir Osmangazi University, Department of Physical Medicine and Rehabilitation, Eskişehir, Turkey

${ }^{3}$ Eskisehir Osmangazi University, Department of Biostatistics, Eskişehir, Turkey

\section{Abstract}

Introduction: Musculoskeletal-pain and sarcopenia are common in elderly and negatively affect living. The objectives were:1-to evaluate the rate of sarcopenia and sarcopenia-related factors according to the European Working Group on Sarcopenia in Older-People-2-2019 algorithm, 2-to compare the rate of probable sarcopenia, sarcopenia parameters and quality of life according to both presence or absence of chronic musculoskeletal-pain and whether the pain is regional or generalized.

Materials and Method: A total of 249-patients were enrolled in this study. The variables: the demographic data, sarcopenia parameters (SARC-F-questionnaire, hand-grip strength, chair-stand test, gait speed), visual analogue scale-pain and quality of life (short-form-36). The patients were divided into two-groups according to presence and absence of chronic musculoskeletal-pain and the chronic pain was divided into three-groups: according to being generalized or regional (one and more than one-site).

Results: Probable sarcopenia was found in $21.6 \%$ of all patients, while $73.4 \%$ had chronic musculoskeletal-pain. The patients with chronic pain (28.4\%) had a higher-rate of sarcopenia than patients without pain $(3 \%)(p<0.001)$, and all the sarcopenia and quality of life parameters were statistically worse. The rate of sarcopenia, social-function, gait-speed, chair-stand test were found similar in three pain groups, visual analog scale-pain $(p=0.001)$, the most of quality of life-parameters, SARC-F-score $(p<0.001)$ and grip-strength $(p=0.009)$ were significantly better in regional pain in one-site.

Conclusion: Female gender, age, diabetes, chronic obstructive pulmonary disease and hypertension were associated with sarcopenia. The rate of sarcopenia was found higher, the sarcopenia and quality of life parameters were worse in patients with musculoskeletal-pain. Although grip-strength and SARC-F-score were better in regional pain in one-site, the rate of sarcopenia was similar in pain groups according to being regional or generalized, and quality of life was worse in generalized pain.

Key Words: Chronic Pain; Sarcopenia; Quality of Life 


\section{INTRODUCTION}

In recent years, the elderly population has been increasing both in Turkey and worldwide. The prevalence of chronic health problems and sarcopenia increase with global aging. Sarcopenia was initially described as age-related muscle mass loss; however, it was later redefined as a dysfunction of the skeletal muscles related with factors such as age, physical inactivity, and nutrition problems. Also, sarcopenia causes disability, morbimortality, and decreases the quality of life (1).

Diagnosing sarcopenia varies due to the different methodologies practiced worldwide. The European Working Group on Sarcopenia in Older People (EWGSOP) 2010 criteria reported a consensus approach (EWGSOP1) which identify sarcopenia based on the presence of low muscle strength or low physical performance in addition to low muscle mass (1). The EWGSOP1 was recently revised in 2019. The revised EWGSOP2 consensus described muscle strength as the key parameter of sarcopenia, with low muscle mass as a diagnostic confirmation of sarcopenia, and poor physical performance as an indication of severe sarcopenia. The EWGSOP2 algorithm recommends the first-line use of the SARC-F questionnaire to identify sarcopenia cases. If results were negative, patients were considered as no sarcopenia; whereas positive results indicate the assessment of muscle grip strength with the use of the grip strength and the chair stand test. If muscle strength were normal, patients were considered as no sarcopenia; but if it were low, sarcopenia probable was considered and muscle mass measurements would be needed to confirm diagnosis. In general, EWGSOP2 recommends that after a positive SARC-F questionnaire response, low muscle strength is sufficient to indicate the assessment of the causes and to start intervention in clinical practice. The first recommended test grip strength which is associated with strength loss of other body parts such as the arm and leg. The other recommended test includes the chair stand test which assesses both endurance and strength (2).

The results of prevalence studies on sarcopenia varied from 15 to $50 \%$, which differed according to age, gender, geographic variables and diagnostic methodologies. Almost all of them were performed based on the EWGSOP1 criteria. The loss of muscle strength rises from 20 to $40 \%$ between the 6th and 7th decades (3). According to the EWGSOP1 criteria, the prevalence of sarcopenia reported by a Turkish study was 5.2\% (4).

Apart from increased sarcopenia rate, chronic musculoskeletal problems also increase with age. In the literature, there are only a few studies on the relationship between chronic pain and sarcopenia or sarcopenia parameters, and their results are different and conflicting (5-7). To the best of our knowledge, this is the first study which evaluated the relationship between sarcopenia, chronic musculoskeletal pain and quality of life according to the EWGSOP2 algorithm.

The aims of this study were: 1 - to evaluate the rate of probable sarcopenia and sarcopenia-related factors according to the European Working Group on Sarcopenia in Older People 2 algorithm, 2- to compare the rate of probable sarcopenia, sarcopenia parameters and quality of life according to both presence or absence of chronic musculoskeletal pain and whether the musculoskeletal pain is regional or generalized.

\section{MATERIALS AND METHOD}

This was a single centre, cross-sectional study carried out between July-August 2020. A total of 249 patients (181 females and 68 males) who were admitted to the physical medicine and rehabilitation clinic were enrolled into this study. Inclusion criteria was being 60 years or older. Patients with Alzheimer's disease, Parkinson's disease, cerebrovascular stroke and other neurological disorders, active cancer, acute/subacute pain, active infection, active arthritis, and impaired cognitive status, as well as 
immobilised patients were excluded from the study.

A detailed anamnesis was carried out on age, gender, weight, height, educational status, working status, family status, the presence of concurrent diseases such as diabetes mellitus, hypertension, chronic obstructive pulmonary disease (COPD), cane usage and chronic musculoskeletal pain. Chronic musculoskeletal pain was defined as persistent pain lasting more than 3 months. Detailed musculoskeletal examinations were carried out by a physical medicine and rehabilitation specialist to identify the source of musculoskeletal pain. Patients with chronic musculoskeletal pain were divided into three groups; patients with musculoskeletal pain in one part of the musculoskeletal system were accepted as "regional pain in one site" (Group 1), patients with regional musculoskeletal pain in more than one site were accepted as "regional pain in more than one site" (Group 2). Patients with pain in four of five regions (left and right upper region, left and right lower region and axial region) was accepped as "generalized pain" (Group 3). Jaw, chest and abdominal pain are not accepted as generalized pain. The visual analogue scale (VAS) was used for measuring general body pain severity, which was assessed from 0 (no pain) to 10 (worst possible pain) (8).

Short Form 36 (SF-36) was used for quality of life assessment. With 36 items, it evaluates physical function, physical role, emotional role, energy, bodily pain, emotional wellbeing, general health, social function and health change. Each scale ranges from 0 (poor health) to 100 (perfect health) (9).

The SARC-F scale evaluates strength, ambulation, rising from a chair, stair climbing, and history of falls. A score lower than 4 was defined as normal. Low gait speed was defined as walking at $\leq 0.8 \mathrm{~m} / \mathrm{s}$ (2). The chair stand test measures the time taken for 5 rounds of rising from the sitting position without using arms, and was defined as low when the time taken was $>15 \mathrm{~s}$. Grip strength was measured with a hand-held dynamometer (Baseline, White Plains,
New York, USA), and the cut-off thresholds were 32 $\mathrm{kg}$ for males and $22 \mathrm{~kg}$ for females (10). Probable sarcopenia was defined according to the EWGSOP2 algorithm as having a low SARC-F score with low muscle strength (grip strength/chair stand test) (2).

Written informed consent was obtained from all patients. Ethics approval was received from the local ethics committee 12/05/20-38 (25403353-050.99E.51559).

\section{STATISTICAL ANALYSIS}

The distribution of each continuous variable was tested for normality using the Shapiro-Wilk test. Non-normally distributed variables were compared using the Mann-Whitney $U$ test and Kruskal-Wallis test ve Bonferroni Post hoc Test are expressed as median values (25\%-75\%). Categorical variables are expressed as frequencies and percentages, and were compared using the Chi-square test. The Spearman correlation coefficient was used as the correlation analysis (a coefficient of $<0.1$ : negligible correlation, 0.1-0.39: weak correlation, 0.4-0.69: moderate correlation, 0.7-0.89: strong correlation, $\geq 0.9$ : very strong) (11). A p-value of $<0.05$ was considered significant. All analyses were performed using the SPSS version 22.0 software (SPSS Inc., Chicago, IL, USA).

\section{RESULTS}

A total of 249 patients (181 females, 68 males) (mean age, $67.6 \pm 6.34$ years) were enrolled. Probable sarcopenia was identified in 54 (21.6\%) patients. Age was found to be higher in probable sarcopenia patients than those without sarcopenia. Although the number of females was higher in both groups, significantly higher rates of probable sarcopenia were found in females than males. In patients without chronic diseases, frequency of probable sarcopenia was found to be significantly lower than in those with concurrent chronic diseases. In terms of distribution of chronic diseases, sarcopenia rate was 
higher in patients with diabetes mellitus, COPD, hypertension and knee prosthesis. Among the probable sarcopenia patients, the rate of working patients was lower, while the rates of retired patients, those living with extended family, and cane usage were higher (Table 1).
Probable sarcopenia was found to be higher in females (25.9\%) than in males (10.2\%). (Table 1). Sarcopenia rate as well as all quality of life and sarcopenia parameters were found to be significantly different between females and males. In females, the VAS-pain score, SARC-F score, and the duration

Table 1. Comparison of demographic characteristics between sarcopenia probable and no sarcopenia patients

\begin{tabular}{|c|c|c|c|}
\hline & $\begin{array}{l}\text { No sarcopenia } \\
\quad(n=195)\end{array}$ & $\begin{array}{l}\text { Sarcopenia probable } \\
(n=54)\end{array}$ & $p$ value \\
\hline Age (median \%25-\%75) & $66.0(62.0-70.0)$ & $69.5(64.7-77.0)$ & $<0.001$ \\
\hline Gender (female/male) n (\%) & 134 (68\%) / 61 (31\%) & 47 (87\%) / 7 (12.9\%) & 0.008 \\
\hline Body Mass Index & $29.6(26.7-32.8)$ & $29.5(27.2-33.0)$ & 0.853 \\
\hline Any other diseases n (\%) & 160 (82.0\%) /35 (17.9\%) & 51 (94.4\%) / 3 (5.5\%) & 0.025 \\
\hline DM & $60(30.7 \%)$ & $26(48.1 \%)$ & 0.017 \\
\hline Cardiac Disease & 38 (19.4\%) & $13(24.0)$ & 0.460 \\
\hline COPD & $21(10.7 \%)$ & $12(22.2 \%)$ & 0.028 \\
\hline Hipertansion & $93(47.6 \%)$ & 37 (68.5\%) & 0.007 \\
\hline Thyroid disease & $19(9.7 \%)$ & $3(5.5 \%)$ & 0.337 \\
\hline Knee prothesis & $14(7.1 \%)$ & $9(16.6 \%)$ & 0.034 \\
\hline Low back surgery & $12(6.1 \%)$ & $4(7.4 \%)$ & 0.740 \\
\hline \multicolumn{4}{|l|}{ Working status n (\%) } \\
\hline Working & 10 (19.5\%) & $2(3.7 \%)$ & \multirow{3}{*}{0.039} \\
\hline Not working & $102(52.3 \%)$ & $12(22.2 \%)$ & \\
\hline Retired & $83(42.5 \%)$ & 40 (74.0\%) & \\
\hline \multicolumn{4}{|l|}{ Educational status n (\%) } \\
\hline Primary school and lower & $167(85.6 \%)$ & 51 (94.4\%) & \multirow{3}{*}{0.148} \\
\hline High school & $10(5.1 \%)$ & $0(0 \%)$ & \\
\hline University and higher & $18(9.2 \%)$ & $3(5.5 \%)$ & \\
\hline \multicolumn{4}{|l|}{ Family status n (\%) } \\
\hline Alone & $26(13.3 \%)$ & 7 (12.9\%) & \multirow{3}{*}{0.047} \\
\hline Nuclear family & 133 (68.2\%) & 28 (51.8\%) & \\
\hline Extended family & $36(18.4 \%)$ & $18(33.3 \%)$ & \\
\hline Cane usage n (\%) & $15(7.6 \%)$ & $20(37.0 \%)$ & $<0.001$ \\
\hline
\end{tabular}

(DM: Diabetes mellitus, COPD: Chronic obstuctive pulmonary disease) 
of chair stand test were higher, while quality of life dimentions, gait speed, and grip strength were lower (Table 2).

All areas of quality of life (SF-36) significantly correlated with VAS-pain scores (weak to moderate correlation) and all sarcopenia parameters (with SARC-F: moderate to strong correlation, with gait speed, grip strength, chair stand test: weak to moderate correlation) $(p<0.001)$, and VAS-pain scores significantly correlated with all sarcopenia parameters (with SARC-F: moderate correlation, with gait speed, grip strength and chair stand test: weak correlation) $(p<0.001)$ (Table 3$)$.

The average age was similar between the patients with $(n=183)$ and without pain $(n=66)(p=0.055)$. The rate of probable sarcopenia was significantly higher in the patients with chronic pain than the patients without pain $(p<0.001)$. Furthermore, all the short form-36 subgroups and the sarcopenia parameters were worse in the patients with chronic pain than the patients without pain (Table 4).

When the patients with chronic pain were evaluated according to having regional [in one $(n=71)$ or more sites $(n=67)]$ or generalized pain $(n=45)$; the average age was found similar between them $(p=0.227)$. The rate of probable sarcopenia, social function dimention of quality life, two of the sarcopenia paremeters (gait speed and chair stand test) was found similar between three groups. The comparison of group 1 and 2 showed that, VASpain $(p=0.002)$, physical function $(p=0.026)$, role physical $(p=0.001)$, role emotional $(p=0.017)$, ener-

Table 2. Comparison of sarcopenia and quality of life parameters according to gender

\begin{tabular}{|c|c|c|c|}
\hline & $\begin{array}{c}\text { Female } \\
\text { median }(\% 25-\% 75) \\
(n=181)\end{array}$ & $\begin{array}{c}\text { Male } \\
\text { median }(\% 25-\% 75) \\
(n=68)\end{array}$ & $p$ value \\
\hline Age & $66.0(62.5-70.0)$ & $67(63-72)$ & 0.357 \\
\hline \multicolumn{4}{|l|}{ SF-36 subgroups } \\
\hline Physical function & $50(30-70)$ & $80(60-95)$ & $<0.001$ \\
\hline Role physical & $50(0-100)$ & $100(50-100)$ & $<0.001$ \\
\hline Role emotional & $33.3(0-66.7)$ & $33.3(66.7-100)$ & $<0.001$ \\
\hline Bodily pain & 55 (32.5-77.5) & $55(25.4-73.7)$ & $<0.001$ \\
\hline Energy & $40(25-52.5)$ & $60(50-75)$ & $<0.001$ \\
\hline Emotional wellbeing & $56(40-72)$ & $72(56-80)$ & $<0.001$ \\
\hline General health & $55(35-70)$ & $65(55-75)$ & $<0.001$ \\
\hline Social function & $100(50-100)$ & $100(78.1-100)$ & 0.003 \\
\hline \multicolumn{4}{|l|}{ Sarcopenia parameters } \\
\hline SARC-F score & $4(2-5)$ & $1(0-4)$ & $<0.001$ \\
\hline Gait Speed & $0.87(0.71-1.0)$ & $1.04(0.9-1.24)$ & $<0.001$ \\
\hline Grip Strength & $22(18-24)$ & $38(32-44)$ & $<0.001$ \\
\hline Chair Stand Test & $14(13-18)$ & $12(10-14)$ & $<0.001$ \\
\hline VAS-pain & $6(5-8)$ & $4(0-6)$ & $<0.001$ \\
\hline
\end{tabular}


Table 3. Correlations of sarcopenia parameters with both quality of life scores and visual analog scale scores

\begin{tabular}{|c|c|c|c|c|c|c|}
\hline & Age & VAS-pain & SARC-F score & Gait speed & Grip strength & $\begin{array}{c}\text { Chair stand } \\
\text { test }\end{array}$ \\
\hline Age & - & $\begin{array}{l}r=-0.096 \\
p=0.140\end{array}$ & $\begin{array}{l}r=0.169 \\
p=0.008\end{array}$ & $\begin{array}{l}r=-0.280 \\
p<0.001\end{array}$ & $\begin{array}{l}r=-0.143 \\
p=0.030\end{array}$ & $\begin{array}{l}r=0.097 \\
p=0.239\end{array}$ \\
\hline VAS-pain & $\begin{array}{l}r=-0.096 \\
p=0.140\end{array}$ & - & $\begin{array}{l}r=0.426 \\
p<0.001\end{array}$ & $\begin{array}{l}r=-0.266 \\
p<0.001\end{array}$ & $\begin{array}{l}r=-0.267 \\
p<0.001\end{array}$ & $\begin{array}{l}r=0.302 \\
p<0.001\end{array}$ \\
\hline \multicolumn{7}{|l|}{ SF - 36 subgroups } \\
\hline Physical function & $\begin{array}{l}r=-0.173 \\
p=0.006\end{array}$ & $\begin{array}{l}r=-0.463 \\
p<0.001\end{array}$ & $\begin{array}{l}r=-0.805 \\
p<0.001\end{array}$ & $\begin{array}{l}r=0.556 \\
p<0.001\end{array}$ & $\begin{array}{l}r=0.523 \\
p<0.001\end{array}$ & $\begin{array}{l}r=-0.502 \\
p<0.001\end{array}$ \\
\hline Role physical & $\begin{array}{l}r=-0.104 \\
P=0.101\end{array}$ & $\begin{array}{l}r=-0.430 \\
p<0.001\end{array}$ & $\begin{array}{l}r=-0.702 \\
p<0.001\end{array}$ & $\begin{array}{l}r=0.465 \\
p<0.001\end{array}$ & $\begin{array}{l}r=0.418 \\
p<0.001\end{array}$ & $\begin{array}{l}r=-0.449 \\
p<0.001\end{array}$ \\
\hline Role emotional & $\begin{array}{l}r=-0.018 \\
p=0.778\end{array}$ & $\begin{array}{l}r=-0.416 \\
p<0.001\end{array}$ & $\begin{array}{l}r=-0.647 \\
p<0.001\end{array}$ & $\begin{array}{l}r=0.392 \\
p<0.001\end{array}$ & $\begin{array}{l}r=0.380 \\
p<0.001\end{array}$ & $\begin{array}{l}r=-0.339 \\
p<0.001\end{array}$ \\
\hline Bodily pain & $\begin{array}{l}r=0.050 \\
p=0.433\end{array}$ & $\begin{array}{l}r=-0.588 \\
p<0.001\end{array}$ & $\begin{array}{l}r=-0.718 \\
p<0.001\end{array}$ & $\begin{array}{l}r=0.447 \\
p<0.001\end{array}$ & $\begin{array}{l}r=0.384 \\
p<0.001\end{array}$ & $\begin{array}{l}r=-0.466 \\
p<0.001\end{array}$ \\
\hline Energy & $\begin{array}{l}r=0.137 \\
p=0.031\end{array}$ & $\begin{array}{l}r=-0.444 \\
p<0.001\end{array}$ & $\begin{array}{l}r=-0.632 \\
p<0.001\end{array}$ & $\begin{array}{l}r=0.447 \\
p<0.001\end{array}$ & $\begin{array}{l}r=0.461 \\
p<0.001\end{array}$ & $\begin{array}{l}r=-0.413 \\
p<0.001\end{array}$ \\
\hline Emotional wellbeing & $\begin{array}{l}r=0.086 \\
p=0.177\end{array}$ & $\begin{array}{l}r=-0.377 \\
p<0.001\end{array}$ & $\begin{array}{l}r=-0.448 \\
p<0.001\end{array}$ & $\begin{array}{l}r=0.327 \\
p<0.001\end{array}$ & $\begin{array}{l}r=0.351 \\
p<0.001\end{array}$ & $\begin{array}{l}r=-0.314 \\
p<0.001\end{array}$ \\
\hline General health & $\begin{array}{l}r=0.020 \\
p=0.751\end{array}$ & $\begin{array}{l}r=-0.422 \\
p<0.001\end{array}$ & $\begin{array}{l}r=-0.628 \\
p<0.001\end{array}$ & $\begin{array}{l}r=0.425 \\
p<0.001\end{array}$ & $\begin{array}{l}r=0.403 \\
p<0.001\end{array}$ & $\begin{array}{l}r=-0.436 \\
p<0.001\end{array}$ \\
\hline Social function & $\begin{array}{l}r=-0.053 \\
p=0.409\end{array}$ & $\begin{array}{l}r=-0.314 \\
p<0.001\end{array}$ & $\begin{array}{l}r=-0.455 \\
p<0.001\end{array}$ & $\begin{array}{l}r=0.300 \\
p<0.001\end{array}$ & $\begin{array}{l}r=0.268 \\
p<0.001\end{array}$ & $\begin{array}{l}r=-0.340 \\
p<0.001\end{array}$ \\
\hline
\end{tabular}

(SF-36: Short Form-36)

gy $(p=0.003)$ dimentions of quality of life was significantly better in group 1 . And the comparison of group 1 and 3 showed that, VAS-pain $(p=0.002)$ all of the qualiy of life dimentions except social function, grip strength $(p=0.002)$ and SARC-F score $(p=0.013)$ was significantly better in group 1 . The comparison of group 2 and 3 showed that only two of the quality of life dimentions [emotional wellbeing $(p=0.002)$ and bodily pain $(p=0.009)]$ were better in group 2 (Table 5).

\section{DISCUSSION}

This study described both the rate of probable sarcopenia, the factors associated with sarcopenia and the relation between sarcopenia, chronic musculoskeletal pain and quality of life in an outpatient clinic using the EWGSOP2 recommended diagnostic algorithm. And found a strong relationship between probable sarcopenia, chronic musculoskeletal pain and poor quality of life.

Sarcopenia can be screened using dual X-ray absorptiometry, bioimpedance analysis, computerised tomography, and magnetic resonance imaging (2). These procedures require time for both patients and hospital personnel. By considering the economic costs as well, there is doubt that these tests add extra value to the diagnosis of sarcopenia. In clinical practice, EWGSOP2 suggests that the SARC-F 
Table 4. The comparison of the quality of life, sarcopenia parameters and the rate of probable sarcopenia between patients with chronic pain and patients with no chronic pain

\begin{tabular}{|c|c|c|c|}
\hline & $\begin{array}{c}\text { Chronic pain } \\
(n=183) \\
\text { median }(\% 25-\% 75)\end{array}$ & $\begin{array}{c}\text { No pain } \\
(n=66) \\
\text { median }(\% 25-\% 75)\end{array}$ & $p$ value \\
\hline \multicolumn{4}{|l|}{ SF-36 subgroups } \\
\hline Physical function & $50(30-70)$ & 85 (65-95) & $p<0.001$ \\
\hline Role physical & $50(0-75)$ & $100(75-100)$ & $p<0.001$ \\
\hline Role emotional & $33.3(0-66.7)$ & $66.7(66.7-100)$ & $p<0.001$ \\
\hline Bodily pain & $45(32.5-67.5)$ & $100(90-100)$ & $p<0.001$ \\
\hline Energy & $40(25-50)$ & $60(50-76.2)$ & $p<0.001$ \\
\hline Emotional wellbeing & $56(40-68)$ & $76(64-80)$ & $p<0.001$ \\
\hline General health & $55(35-65)$ & $70(60-80)$ & $\mathrm{p}<0.001$ \\
\hline Social function & $100(50-100)$ & $100(87.5-100)$ & $p<0.001$ \\
\hline \multicolumn{4}{|l|}{ Sarcopenia parameters } \\
\hline SARC-F score & $4(2-5)$ & $1(0-2)$ & $p<0.001$ \\
\hline Gait Speed & $0.84(0.70-1.0)$ & $1.06(0.96-1.25)$ & $p<0.001$ \\
\hline Grip Strength & $22(18-28)$ & $30(24-40)$ & $\mathrm{p}<0.001$ \\
\hline Chair Stand Test & $14(12-18)$ & $12(9.6-14)$ & $p<0.001$ \\
\hline The rate of sarcopenia $\mathrm{n}(\%)$ & $52(28.4 \%)$ & $2(3 \%)$ & $p<0.001$ \\
\hline
\end{tabular}

(SF-36: Short Form-36)

questionnaire and muscle strength measurement are sufficient to indicate the assessment of causes of sarcopenia and the start of intervention.

In our study, patients with positive SARC-F questionnaire results and low muscle strength were described as probable sarcopenia and they accounted for $21.6 \%$ of all patients. Furthermore, sarcopenia was found to be associated with age, female gender, chronic diseases, and chronic musculoskeletal pain. In another study from Turkey, Erkoyun et al. investigated sarcopenia risk according to EWGSOP1, and reported a prevalence of having both low grip strength and low gait speed to be $30.1 \%$. It was also reported that the risk of sarcopenia increases with age (12). Consistent with studies from other countries and Turkey $(4,12)$, sarcopenia parameters correlated with age. Muscle mass loss is associated with decreased hormone levels, increased proinflammatory cytokines and reactive oxygen radicals with aging (13). Early detection of sarcopenia is important; resistance exercises, increased protein intake, vitamin D and omega 3 supplementations are recommended in elderly (14). In terms of quality of life, age only correlated with physical function and energy parameters, which are expected to decrease with age. However, other parameters did not correlate with age. Consistent with the results from previous studies (15), aging did not decrease quality of life, suggesting that it is possible to achieve long periods of good quality of life with physical activities such as walking (16). 
Table 5. The comparison of the VAS-pain scores, quality of life, sarcopenia parameters and the rate of probable sarcopenia between the patients with regional pain in one site, the patients with regional pain in more than one site and the patients with generalized pain

\begin{tabular}{|c|c|c|c|c|c|}
\hline \multirow[b]{2}{*}{ Variables } & \multicolumn{3}{|c|}{$\begin{array}{c}\text { Chronic pain } \\
(n=183) \\
\text { No pain }\end{array}$} & \multirow[b]{2}{*}{$p$ value } & \multirow{2}{*}{$\begin{array}{l}\text { Bonferroni } \\
\text { Post hoc Test }\end{array}$} \\
\hline & $\begin{array}{c}\text { Group } 1 \\
\text { Regional pain in one } \\
\text { side }(n=71) \\
\text { median }(\% 25-\% 75)\end{array}$ & $\begin{array}{c}\text { Group } 2 \\
\text { Regional pain in more } \\
\text { than one side }(n=67) \\
\text { median }(\% 25-\% 75)\end{array}$ & $\begin{array}{c}\text { Group } 3 \text { Generalized } \\
\text { Pain }(n=45) \\
\text { median }(\% 25-\% 75)\end{array}$ & & \\
\hline VAS-pain & $6(5-8)$ & $7(6-8)$ & $8(6-8)$ & 0.001 & $\begin{array}{l}\text { 1-2: } 0.002 \\
\text { 1-3: } 0.002\end{array}$ \\
\hline \multicolumn{6}{|l|}{ SF-36 subgroups } \\
\hline Physical function & $60(45-80)$ & $45(25-60)$ & $35(15-62.5)$ & 0.001 & $\begin{array}{l}1-2: 0.026 \\
1-3: 0.001\end{array}$ \\
\hline Role physical & $50(25-100)$ & $25(0-75)$ & $25(0-50)$ & $<0.001$ & $\begin{array}{c}1-2: 0.001 \\
1-3:<0.001\end{array}$ \\
\hline Role emotional & $66.7(33.3-66.7)$ & $33.3(0-66.7)$ & $0(0-33.3)$ & $<0.001$ & $\begin{array}{l}\text { 1-2: } 0.017 \\
\text { 1-3: } 0.001\end{array}$ \\
\hline Bodily pain & $55(32.5-67.5)$ & $45(32.5-67.5)$ & $42.5(22.5-55)$ & $<0.001$ & $\begin{array}{c}1-3:<0.001 \\
2-3: 0.009\end{array}$ \\
\hline Energy & $45(30-65)$ & $40(25-50)$ & $25(20-35)$ & $<0.001$ & $\begin{array}{c}1-2: 0.003 \\
1-3:<0.001\end{array}$ \\
\hline Emotional wellbeing & $60(40-76)$ & $56(44-72)$ & $40(28-60)$ & $<0.001$ & $\begin{array}{c}\text { 1-3: }<0.001 \\
\text { 2-3: } 0.002\end{array}$ \\
\hline General health & $60(50-70)$ & $55(35-70)$ & $40(25-50)$ & $<0.001$ & 1-3: 0.003 \\
\hline Social function & $100(50-100)$ & $100(50-100)$ & $50(25-100)$ & 0.052 & \\
\hline \multicolumn{6}{|c|}{ The sarcopenia parameters } \\
\hline SARC-F score & $3(1-5)$ & $4(3-6)$ & $4(3.5-6.5)$ & $<0.001$ & 1-3: 0.013 \\
\hline Gait Speed & $0.9(0.82-1.02)$ & $0.82(0.68-1)$ & $0.77(0.59-1.02)$ & 0.065 & \\
\hline Grip Strength & $24(20-32)$ & $22(16-26)$ & $20.5(16-24.5)$ & 0.009 & 1-3: 0.002 \\
\hline Chair Stand Test & $13(12-16)$ & $15(13-19)$ & $14(12-19.2)$ & 0.459 & \\
\hline The rate of sarcopenia & $15(21.1 \%)$ & $24(35.8 \%)$ & $13(28.9 \%)$ & 0.160 & \\
\hline
\end{tabular}

(SF-36: Short Form-36, VAS-pain: Visual analog scale-pain) 
In our study, both the rate of sarcopenia and quality of life were worse in females than males, with $25.9 \%$ of female but only $10.2 \%$ of males had probable sarcopenia. Although sex-adjusted prevalence varies between countries, sarcopenia and poor quality of life are generally higher in women. Similar to our study, the prevalence of sarcopenia was reported to be $33 \%$ in females and $10 \%$ in males in Spain, a Mediterranean country such as ours (17). Also a study in the US reported that quality of life was clearly lower in women, and explained that this could be due to sociodemographic and socioeconomic differences (18). All these results showed that women have higher risk of sarcopenia and lower quality of life. These consequences may be related to the place of women in society.

Similar to our study, previous studies have shown a synergistic relationship between chronic diseases and sarcopenia. It has been reported that the prevalence of sarcopenia in diabetic patients was 2-3 times higher than non-diabetics (19). Moreover, a decline in grip strength was reported in middle-aged Japanese-American men with diabetes and COPD (20). Similar results were reported in a prevalence study in Turkey, whereby diabetes and hypertension associated with sarcopenia (4).

In previous studies, socio-economic factors were not found to be associated with sarcopenia (21). In our study, retired patients were found to be at a higher risk for sarcopenia. Retirement may be associated with reduced physical activity, resulting in muscle strength decrease. Family type was also found to be associated with sarcopenia; however, no such association was found with education status. An extended family as a risk factor for sarcopenia was unexpected, but it is possible that patients who are unable to meet their own needs due to sarcopenia mostly require the care of an extended family.

In our study, all of the sarcopenia parameters were better and the sarcopenia rate was significantly higher in patients without chronic pain than those with chronic musculoskeletal pain. Although grip strength and SARC-F score were better in patients with regional pain in one site than patients with generalized pain, the rate of sarcopenia was similar between these groups. Chronic musculoskeletal pain was found to be associated with the higher rate of sarcopenia however being regional or generalized pain does not appear to be associated with high sarcopenia rate. Besides, the severity of pain correlated with SARC-F questionnaire and most of the quality of life parameters moderately. The prevalence of chronic musculoskeletal pain is approximately $50 \%$ in elderly people in the community (22), but was reported in $73.4 \%$ of all our patients. This rate is high, yet expected since the study was conducted in an outpatient clinic, not in the community. There are, however, conflicting results regarding the relationship between chronic pain and sarcopenia. Similar to our study, Zanin et al. found an association between sarcopenia and chronic pain in 79 institutionalised elderly women with positive SARC-F questionnaire results for sarcopenia (5). Likewise, Hicks et al. reported a relationship between higher low back pain severity and lower trunk muscle attenuation (6). In Japan, Tanishima et al. defined sarcopenia patients as being over 40 years old, having low grip strength, and/or low gait speed with low muscle mass according to the Asian Working Group for Sarcopenia, and low back pain prevalence was similar across the sarcopenia, pre-sarcopenia, and normal groups; but similar to our study, VAS-pain scores were found to be higher in the sarcopenia group (23). On the other hand, a study in Brazil reported no association between hand grip strength and chronic pain (24), and a Japanese study reported no association between appendicular muscle mass and back/low back pain (7). Chronic musculoskeletal problems have been reported to be associated with decreased activities of daily living and quality of life. As the intensity of pain increases with static contraction of muscles, the tendency for patients to protect the pain area increases. Prolonged periods without treatment would hence result in the 
loss of muscle strength (25). In light of this, chronic musculoskeletal pain exacerbates sarcopenia progression by inhibiting physical activity.

Our study showed that quality of life was poor in patients with chronic musculoskeletal pain than without pain. Besides, patients with generalized pain had worse quality of life than patients with regional pain in one site. This is an expected result, the relation between pain and quality of life in elderly was reported in earlier studies (26).

There were several limitations in this present study; among them being that a definitive diagnosis of pain such as disc herniation, spinal stenosis, and osteoarthritis was not adopted. However, to the best of our knowledge, our study is the first to find the relationship between sarcopenia and chronic pain according to the EWGSOP2 algorithm. Our results can not be generalised to general population. Further studies are needed to investigate both sarcopenia prevalence in general population in Turkey and the relationship between sarcopenia and definite musculoskeletal conditions such as osteoarthritis.

Our study results showed that; the rate of probable sarcopenia was $21.6 \%$ of all patients, and also

\section{REFERENCES}

1. Cruz-Jentoft AJ, Baeyens JP, Bauer JM, et al. Sarcopenia: European consensus on definition and diagnosis: report of the European Working Group on Sarcopenia in Older People. Age Ageing 2010; 39: 412-23. (PMID: 20392703)

2. Cruz-Jentoft AJ, Bahat G, Bauer J, et al. Sarcopenia: revised European consensus on definition and diagnosis. Age Ageing 2019; 48(1):16-31. (PMID: 30312372)

3. Castillo EM, Goodman-Gruen D, Kritz-Silverstein D et al. Sarcopenia in elderly men and women. The Rancho Bernardo Study. Am J Prev Med 2003;25:226-31. (PMID: 14507529)

4. Simsek H, Meseri R, Sahin S, et al. Prevalence of sarcopenia and related factors in community-dwelling female gender, age, and chronic diseases, especially diabetes, COPD and hypertension, are associated with sarcopenia. The sarcopenia parameters and quality of life of the patients with chronic musculoskeletal pain were worse than patients without pain. Furthermore, the rate of probable sarcopenia was found higher in patients with chronic musculoskeletal pain than patients without pain. Although quality of life and some of the sarcopenia parameters of the patients with generalized pain were worse than patients with regional pain in one site, the rate of sarcopenia was similar between patients with generalized pain, regional pain in one site and more than one site. In conclusion, a strong relationship between sarcopenia, chronic musculoskeletal pain and poor quality of life was found. Also only age and gender were the non-preventable risk factors for sarcopenia, while chronic diseases and musculoskeletal pain were preventable. To prevent sarcopenia, early and effective treatment for chronic diseases and musculoskeletal problems represent an important target.

\section{Conflict of Interest}

All authors declare no conflict of interest.

elderly individuals. Saudi Med J 2019;40(6):568-74. (PMID: 31219491)

5. Zanin C, Candido JB, Jorge MSG, et al. Sarcopenia and chronic pain in institutionalized elderly women. BrJP 2018;1(4):288-92. (DOI: 10.5935/2595-0118.20180055)

6. Hicks GE, Simonsick EM, Harris TB, et al. Trunk muscle composition as a predictor of reduced functional capacity in the health, aging and body composition study: the moderating role of back pain. J Gerontol A Biol Sci 2005;60(11):1420-4. (PMID: 16339328)

7. lizuka Y, lizuka H, Mieda T, et al. Association between neck and shoulder pain, back pain, low back pain and body composition parameters among the Japanese general population. BMC Musculoskelet. Disord. 2015;16(1): 333. (PMID: 26537689) 
8. Gallagher EJ, Liebman M, Bijur PE. Prospective validation of clinically important changes in pain severity measured on a visual analog scale. Ann Emerg Med 2001;38:633-8. (PMID: 11719741)

9. Kocyigit $\mathrm{H}$, Aydemir O, Fisek G, et al. Validity and reliability of Turkish version of short form 36: a study of patients with rheumatoid disorder. J Drug Ther 1999;12:102-6. (in Turkish)

10. Bahat G, Tufan A, Tufan F, et al. Cut-off points to identify sarcopenia according to European Working Group on Sarcopenia in Older People (EWGSOP) definition. Clin nutr 2016; 35(6):1557-63. (PMID: 26922142)

11. Schober P, Boer C, Schwarte LA. Correlation coefficients: appropriate use and interpretation. Anesth. Analg. 2018;126(5):1763-8. (PMID: 29481436)

12. Erkoyun E, Ucku R. The prevalence of sarcopenia risk and associated factors in patients aged 65-79 years living in a district of Izmir province of Turkey. Turk J Phys Med Rehabil 2020; 66(1):10-16. (PMID:32318669)

13. Discigil, G, Sokmen UN. Sarcopenia in the elderly. J Turk Family Physician 2017; 8(2). (In Turkish)

14. Eyigor S, Kutsal YG. Sarcopenia: Again and updated. Turkish J Geriatrics 2020; 23(1):1-7. (DOI: 10.31086/ tjgeri.2020.131)

15. Netuveli G, Blane D. Quality of life in older ages. Br. Med. Bull 2008; 85(1):113-26. (PMID: 16537355)

16. Sekerci YG, Bicer EK. The Effect of Walking Exercise on Quality of Life and Sleep in Elderly Individuals: Randomized Controlled Study. Turkish J Geriatrics 2019; 22(4):443-53. (DOI:10.31086/tjgeri.2020.123)

17. Masanes F, Culla A, Navarro-Gonzalez M, et al. Prevalence of sarcopenia in healthy community-dwelling elderly in an urban area of Barcelona (Spain). J Nutr Health Aging 2012; 16: 184-7. (PMID: 22323356)

18. Cherepanov D, Palta M, Fryback DG, et al. Gender differences in health-related quality-of-life are partly explained by sociodemographic and socioeco- nomic variation between adult men and women in the US: evidence from four US nationally representative data sets. Qual Life Res 2010; 19(8):1115-24. (PMID: 20496168)

19. Koo BK, Roh E, Yang YS, et al. Difference between old and young adults in contribution of $\boldsymbol{\beta}$-cell function and sarcopenia in developing diabetes mellitus. J. Diabetes Investig 2016;7:233-40. (PMID: 27042276)

20. Rantanen T, Masaki K, Foley D, et al. Grip strength changes over 27 yr in Japanese-American men. J Appl Physiol 1998;85:2047-53. (PMID: 9843525)

21. Legrand D, Vaes B, Mathei $C$, et al. The prevalence of sarcopenia in very old individuals according to the European consensus definition: insights from the BELFRAIL study. Age Ageing 2013; 42: 727-34. (PMID: 24014657)

22. Pereira LV, De Vasconcelos PP, Souza LA, et al. Prevalence and intensity of chronic pain and self-perceived health among elderly people: a population-based study. Rev Lat Am Enfermagem 2014;22(4):662-9. (PMID:25296151)

23. Tanishima $S$, Hagino $H$, Matsumoto $H$, et al. Association between sarcopenia and low back pain in local residents prospective cohort study from the GAINA study. BMC Musculoskelet. Disord. 2017; 18(1):1-6. (PMID:29141602)

24. Ribeiro DS, Garbin K, Jorge MSG, et al. Prevalence of chronic pain and analysis of handgrip strength in institutionalized elderly. BrJP 2019; 2(3):242-6. (DOI: 10.5935/2595-0118.20190043)

25. Falla D, Farina D, Dahl MK, et al. Muscle pain induces task-dependent changes in cervical agonist/antagonist activity. J Appl Physiol 2007;102(2):601-9. (PMID: 17038492)

26. Gokkaya NKO, Kutsal YG, Borman P, et al. Pain and quality of life (OoL) in elderly: The Turkish experience. Arch Gerontol Geriatr 2012;55(2):357-62. (PMID: 22104759) 